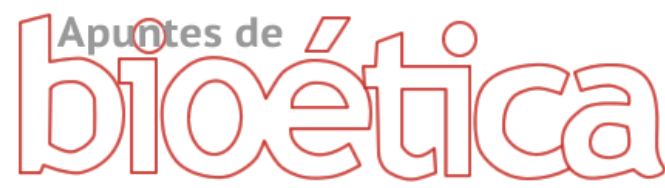

https://doi.org/10.35383/apuntes. v1i1.189

\title{
Directrices de Salud para implementar programas de educación de la sexualidad, con visión personalista, para adolescentes - Perú
}

\author{
Rocío Miranda Palacios ${ }^{1}$
}

\section{INFORMACIÓN DEL ARTÍCULO RESUMEN}

Recibido el 26 de setiembre de 2018

Aceptado el 20 de noviembre de 2018

\section{Palabras claves:}

Ética,

Adolescentes,

Sexualidad
Educación de la sexualidad,

La realidad nos muestra que la educación en la sexualidad está siendo abordada de forma deficiente ya sea porque es reducida a la simple genitalidad, dándole un enfoque biológico o cultural o porque los gobiernos pretenden impartir a los jóvenes en los colegios una educación sexual meramente instrumental trayendo como consecuencia una banalización de la sexualidad en los adolescentes. El objetivo de la presente investigación es establecer directrices de salud para la implementación de programas de educación de la sexualidad, con visión personalista, para adolescentes en el Perú, basada en la complementariedad o integridad.

Health Guidelines with a personalist vision to implement sexuality education programs for adolescents - Peru

\section{ABSTRACT}

Keywords:

Adolescents

Sexuality education,

Sexuality

Reality shows us that sexuality education is being addressed poorly either because it is reduced to mere genitality, giving a biological or cultural approach, or because governments aim to provide young people in schools a purely instrumental sex education bringing as a result a trivialization of sexuality in adolescents. The aim of this research is to establish guidelines for the implementation of health education programs sexuality, with personalist vision for adolescents in Peru, based on complementarity or integrity.

\footnotetext{
1 Licenciada en Obstétrica, Maestra en Persona, Matrimonio y Familia. Actualmente labora en el Hospital San Vicente de Paúl (Camerún). Email rociomiranda13@yahoo.es, mirandapalacios.rocio@gmail.com ORCID: https://orcid.org/0000-0001-5439-1025
} 


\section{Introducción}

Preparar a los adolescentes para una exitosa transición a la adultez siempre ha sido uno de los mayores retos que la humanidad ha debido enfrentar. La sexualidad constituye un tema central para el desarrollo de toda personalidad; sobre todo de los adolescentes. En este tema los padres y la familia desempeñan una función vital en la formación de la comprensión respecto de la identidad sexual de sus hijos. Lamentablemente dicho compromiso no está siendo asumido adecuadamente por estos y es irrogada por el Estado; esto conlleva a que esta educación sea realizada de forma deficiente.

Son muy pocos los adolescentes que reciben una adecuada educación de su sexualidad. Al respecto, la Organización de las Naciones Unidas para la Educación de la Ciencia y la Cultura (UNESCO), afirma que: (...) personas jóvenes, llegan a la adultez con mensajes contradictorios y confusos sobre la sexualidad, viéndose reflejado en la iniciación temprana de la actividad sexual por parte de los jóvenes o la tendencia actual de postergar el matrimonio, prolongando de esa forma el período de tiempo comprendido entre el inicio de su actividad sexual y la formalización de sus relaciones de pareja (UNESCO, 2014).

Además de lo manifestado, la falta de conocimiento y educación de sexualidad en los adolescentes hacen que estos tomen decisiones y se expongan a las diferentes conductas de riesgo que predispone a estar vulnerables a la alteración de su salud, constituyendo un problema de salud pública. Por otro lado el aumento de embarazos en adolescentes, abortos provocados, embarazos no deseados o no planificados, sigue siendo también un problema de salud pública, que afecta directamente a la mujer adolescente. Esto se ve reflejado en las estadísticas presentadas por el MINSA en el año 2014.

Dicha realidad nos muestra la necesidad de brindar a los adolescentes una educación adecuada que les permitan hacer elecciones responsables en sus vidas en un tema de creciente preocupación en todos los países, como es el tema de la sexualidad humana. Descrita la realidad anterior es que nos planteamos la siguiente interrogante como tema de estudio: ¿Cuáles serán las directrices de salud para la implementación de programas de educación de la sexualidad, con visión personalista, para adolescentes en Perú?

Nuestra investigación tiene por finalidad otorgar directrices de salud para la implementación de programas de educación en sexualidad para adolescentes en el Perú; pretendiendo dar un nuevo enfoque de forma integral y una orientación adecuada respecto a la educación en sexualidad basada en la persona en su totalidad.

Para esto proponemos como objetivo general: Establecer directrices de salud para la implementación de programas de educación de la sexualidad, con visión personalista, para adolescentes en Perú y como objetivo específico proponemos: Argumentar la necesidad de un enfoque integral de salud basada en la visión personalista para la educación de la sexualidad. Para ello se tiene como pilares de esta propuesta: la castidad y la complementariedad; así como también considerar la realidad en la que se desenvuelve y no reducirla a la descripción de peligros que acechan; por el contrario "debemos hacer hincapié en describir sus ventajas y la felicidad de quienes, desde un concepto de sexualidad respetuoso con la naturaleza de las personas son capaces de amar mejor" (De Irala, 2012, p. 8). 
La importancia del presente trabajo se centra en dar respuestas a la interrogante planteada, determinando si existe o no la necesidad de dar directrices de salud para la implementación de programas educación de la sexualidad, con visión personalista, en adolescentes en el Perú; ayudando a la mejora de la salud pública a nivel nacional.

\section{Metodología}

La presente investigación es de tipo cualitativa, descriptiva e interpretativa; de corte humanista, inductiva con una perspectiva integral de la persona, en especial del adolescente, que es sujeto de la investigación. Dentro del abordaje metodológico, las técnicas utilizadas son las notas de resumen que permitieron en forma breve concretar la información recabada, esquemáticamente por temas. También están las notas de análisis y comentarios de documentos que por inducción se categorizó la información para el análisis y deducir lo requerido; finalmente los resultados de la búsqueda electrónica en internet fueron diversos y de calidad garantizándose el valor científico. Como instrumento de recolección de datos está la observación documental que interactúa en la realidad; trabajándose también con notas bibliográficas, textuales, de resumen y de síntesis, las cuales permitieron recoger, almacenar, organizar y presentar la información extraída de las fuentes tales como libros, revistas, entre otros. El procedimiento se realizó mediante la recopilación del material bibliográfico, que permitió establecer los fundamentos para el desarrollo de nuestro objeto de investigación.

\section{Resultados, análisis y discusión}

\section{Necesidad de la Educación de la Sexualidad}

Pocas personas que reciben una adecuada preparación para la vida y para educar integralmente su sexualidad y/o aceptar la tarea de proporcionar una educación en sexualidad clara, informada, sustentada en hechos científicos e inspirada en los valores universales. 4 La mayor parte de autores utilizan el término educación sexual para referirse a la educación de la sexualidad; término que consideramos no son iguales. En este sentido Víctor García (2012) afirma que: "debería llamarse educación de la sexualidad en razón de su vinculación a todas las manifestaciones de la persona humana" (p. 17).

Al inicio la educación sexual comenzó dando respuesta a los diferentes problemas de la sociedad relacionados con la salud pública, en razón de ello, la educación sexual se centró en la realización de programas dedicados a impartir conocimientos acerca de la reproducción humana, anticoncepción, enfermedades de transmisión sexual, aborto, etc, es decir sólo estaba orientada a un sentido biológico. Actualmente esta forma de pensamiento sigue prevaleciendo pero no se puede dejar de pensar que la sexualidad está estrechamente vinculada con la dignidad de la persona; con un único cimiento sólido para que no sean las leyes externas que prohíban o no sino la convicción interior de cada hombre que se reconozca como persona; que adopte y acepte los riesgos de hoy y los evite educando su sexualidad, es decir una educación de la sexualidad en el ser humano con un enfoque integral (Garcia, 2012). 


\section{Presupuestos para una adecuada educación de la sexualidad}

La sexualidad humana es una dimensión constitutiva de persona humana, porque tiene que ver con su identidad y modo de ser, con la forma que se comunica con los demás, con su desarrollo y crecimiento y con la capacidad de dar vida,implicando todo el ser; por ello hay que educar y formar la sexualidad durante toda la existencia; siendo así, las decisiones iniciales sobre sexualidad de gran importancia porque marcan la vida y el futuro de cada persona; acertar estas decisiones facilita alcanzar la felicidad y una vida plena (De Irala \& Beltramo, 2013). Existe una variedad de presupuestos que se deberían tomar en cuenta cuando se estudia la educación de la sexualidad, nosotros consideramos conveniente que agrupar en dos presupuestos básicos:

- La educación de la castidad

- La enfoque de la complementariedad o de la integridad

\section{A. Educación de la Castidad}

La educación de la sexualidad se concreta en la educación en y para la castidad (Sarmiento, 1999). Por ello, ha de formar parte de esa educación integral, que, "en el marco de una antropología genuina y rica"9, haga descubrir que el auténtico sentido de la libertad en el que consiste la verdadera realización personal en el ejercicio de la sexualidad, "está orientado al don de sí en la comunión" (Sarmiento, 1999) dentro de la apertura hacia el otro.

La formación y educación de la castidad, es el modo concreto de llevar a cabo la verdadera educación de la sexualidad porque implica necesariamente la formación y educación en las demás virtudes, como puede ser entre otras: la templanza, la fortaleza y la prudencia o aquellas que tengan estén relacionadas con las tendencias sexuales (Garcia, 2012).

También la castidad es expresión ordenada de la unidad substancial de la persona humana y representa el verdadero lenguaje del cuerpo humano, suponiendo a la vez la integridad de la misma. Es la virtud que orienta la actividad de la sexualidad hacia su propio bien, integrándolo en el bien de la persona, conforme con la dignidad y observando el orden moral recto; haciendo que el lenguaje de la sexualidad no se degrade y responda a la verdad que está llamado a expresar; impregnando de racionalidad el ejercicio de la sexualidad y así percibir su significado y realizando en toda su verdad e integridad (Garcia, 2012).

La unidad de la persona se opone a todo comportamiento que la pueda lesionar; no tolera ni la doble vida ni el doble lenguaje; entraña la totalidad del don, porque para ser castos no basta con someter las pasiones al dominio de la razón, es necesario que ese dominio consecuencia del señorío sobre uno mismo este al servicio del amor, el cual sólo es verdadero si es total, es decir, si a la persona del otro se le valora por lo que es; exigiendo una relación de donación gratuita y desinteresada. El dominio de sí, propio de la virtud de la castidad, esta ordenado al don de sí mismo (Garcia, 2012).

\section{B. Enfoque de la Complementariedad o de la Integridad}

El fundamento de éste enfoque reside en las categorías de igualdad y diferencia entre varón y mujer, se presupone así en términos muy generales, que ambos son diferentes y a la vez iguales. Son diferentes desde el plano genético, 
endocrinológico, psicológico, anatómico, etc.; dichas diferencias no quiebran la igualdad ontológica, en cuanto son personas poseyendo una igual dignidad ontológica. Así de este modo, hacer esta distinción presupone necesariamente la igualdad (Castilla De Cortazar, 2004).

La categoría de la igualdad entre varón y mujer es un presupuesto incuestionable, incluso para la propia complementariedad. Ambos, varón y mujer, participan de una misma naturaleza y poseen una misión conjunta: la familia y la cultura. Por ello la complementariedad dilucida donde se encuentra la diferencia e inserta la igualdad, de modo que ninguna categoría lesione o le reste su lugar a la otra. Antropológicamente explicable qué y por qué las mujeres son diferentes a los hombres (Haaland Matlary, 2000) y al determinar en qué consiste la diferencia, tendrá que precisar que tiene de cultural y qué de permanente en la condición sexuada, explicando la armonía entre igualdad y diversidad (Castilla De Cortazar, 2004).

Al referirnos al presupuesto de la igualdad ontológica, se sabe que, sea cual sea la diferencia entre hombres y mujeres siempre se presupone la igualdad porque ambos son personas $y$, en consecuencia, poseen el mismo rango ontológico. Por ello, tanto el varón como la mujer, están llamados a ser protagonistas de un progreso equilibrado y justo, que promueva la armonía y la felicidad. Y por el principio de la igualdad, tenemos dos elementos estructurales comunes a varones y mujeres: su dignidad intrínseca y su carácter relacional.

El principio de la dignidad humana intrínseca del ser humano implica el hombre, posee una excelencia o eminencia ontológica, una superioridad, no son sólo animales de una especie superior, sino que pertenecen a otro orden, más eminente o excelente, en razón de lo cual merecen ser considerados personas, (Elosegui Itxaso, 2011) y se presupone la existencia de una naturaleza humana que es común a varones y mujeres; fundamento importante para el reconocimiento de la igualdad.

Otro punto que sustenta la igualdad, el carácter relacional de hombre; el varón y la mujer son seres relacionales. La dimensión de interdependencia es también consustancial a la persona. En realidad, el ser humano es un ser con los demás. La persona es, por constitución, máxima comunicación; la estructura relacional está enclavada en el ser de la persona.

Por ello, cuando se da un enfoque sólo biologicista es justamente denigrado por esencialista o naturalista y el postfeminismo de género puede ser tachado también de reduccionista o "culturalista", en cuanto que ignora que el ser humano es también su cuerpo, su realidad biológica.

Lo que buscamos es hacer compatibles la igualdad y la diferencia entre varón y mujer. Igualdad por su condición de personas e igual dignidad, de la cual deriva la igualdad de derechos; participan de una misma naturaleza humana y una misiónconjunta: la familia y la cultura. La defensa de la igualdad en la dignidad y en los derechos no es obstáculo, para defender al mismo tiempo la diferencia entre varón y mujer, sea a nivel genético, anatómico, hormonal, psicológico, etc.; porque pone sus fundamentos en la realidad de la existencia humana (Aparisi, 2011).

Desde el ámbito científico, analizando el punto de vista biológico la persona existe como varón o mujer; de manera innata y natural, se desarrolla diferenciándose en cuerpo humano 
femenino o masculino; dándose este proceso de dimorfismo desde la gametogénesis. Los gametos que aporta el organismo en la fecundación son esencialmente diferentes, dependiendo si proviene de la mujer o del varón; genéticamente la mujer tiene el cromosoma sexual, pero el cromosoma $\mathrm{X} \circ \mathrm{Y}$ del gameto masculino es el que determina el sexo cromosómico, del nuevo individuo. Dicho sexo cromosómico determina el sexo gonádico y éste el hormonal y demás. Por ello por la condición sexuada de la persona, el pertenecer al sexo masculino o femenino, está determinado y se plasma desde la concepción, a niveles genéticos, gonádicos, hormonales y morfológicos; creciendo y desarrollándose como individuo a partir de lo genético de manera diversa y complementaria; dicha condición sexual, acompaña al ser humano desde su origen y durante toda su existencia (Lucas Lucas, 2008).

El origen y la orientación de la sexualidad se encuentran inscritos en el nivel biológico, pero su naturaleza no se queda en este orden, porque en su maduración y manifestación intervienen factores psicológicos, sociales y culturales como son: la educación, el ambiente familiar, las amistades, etc., que influyen en la persona desde que es concebida, donde nace y crece, ejerciendo su capacidad racional y volitiva, desarrollándose a través del aprendizaje y educación, habiendo de esta manera una presencia y una interacción del individuo sexuado, sea varón o mujer (Lucas Lucas, 2008).

3. Propuesta de directrices de salud para la implementación programas de educación de la sexualidad para adolescentes en Perú

Proponemos una concepción integral de la persona, que no dividan al ser humano, sino que se tenga la capacidad de integrarla, con un carácter unitario, que busque comprender a la persona como una unidad inescindible entre cuerpo y espíritu; entre dimensión corporal y autonomía y racionalidad; entre naturaleza y cultura; entre sexo y género, proponiendo por ello un modelo de la integridad y la complementariedad, (Aparisi, 2011)por existir una gran necesidad de este enfoque adecuado para la educación de la sexualidad para adolescentes; por lo que a continuación damos las directrices de salud a considerar para implementar programas de educación de la sexualidad con visión personalista para adolescentes en Perú:

- Visión integral de la persona y de su sexualidad

- La persona es cuerpo y espíritu

- Importancia de una adecuada educación de la identidad sexual

- Dos modos de ser: masculino y femenino

- Reciprocidad y Complementariedad

- La dimensión de apertura en el varón y en la mujer

\section{Visión integral de la persona y de su sexualidad}

La primera directriz a considerar es que la visión personalista propone una concepción integral de la persona, que no divide al ser humano, sino que busca integrarlo en su carácter unitario. Por ello es muy importante la integración de la sexualidad en la persona, de forma más concreta que afianzando la idea que la persona humana es persona sexuada, distinguiendo el carácter sexual del carácter sexuado de la persona. El carácter sexual nos lleva a un dato netamente biológico, siendo varón o mujer y el carácter sexuado de la persona, nos hace comprender a la persona como ser sexuado, existiendo dos modos de ser 
persona: persona masculina y persona femenina; realidad estructuralmente sexuada y dato que afecta a toda la identidad sexual: se es varón o se es mujer en todas y cada una de sus dimensiones, capacidades y cualidades que configuran la persona (Burgos, 2011).

\section{La persona es cuerpo y espíritu}

Una segunda directriz nos lleva a considerar que el cuerpo y el espíritu constituyen esa totalidad unificada corpóreo - espiritual que es la persona humana, existente como hombre o como mujer, no hay otra posibilidad; el espíritu se une al cuerpo que es masculino o femenino y por la totalidad de la unidad substancial cuerpo y espíritu es totalmente masculino o femenino e inseparable de la persona; por tanto es una persona sexuada y el espíritu humano se orienta a informar al cuerpo que necesariamente es varón o mujer, de allí su fin.

Por tanto la sexualidad no es un simple atributo, es la modalidad substancial del ser de la persona humana, es decir que la sexualidad penetra totalmente la humanidad del varón y de la mujer; la masculinidad y la feminidad va caracterizando y determinando a todos y cada uno de los componentes de esta unidad substancial cuerpo - espíritu que llamamos varón o mujer; afectando la sexualidad el núcleo íntimo de la persona; que siente y se expresa a través de la misma.

\section{Importancia de una adecuada educación de la identidad sexual}

La identidad sexual se configura por la complementariedad entre sexo y género; el sexo, está determinado biológicamente, es lo dado, no es elegible. Sin embargo el género es lo adquirido por medio de factores culturales como la educación, el ambiente, las relaciones sociales, etc.28 Urge una adecuada educación de la sexualidad en adolescentes orientada a que éste conozca su identidad sexual adecuadamente.

La realidad biológica tiene en lo profundo un significado personal, una identidad natural esencial, que permite al ser humano reidentificarle a partir de lo externo; este indicio es muy importante y crucial porque implica la identidad personal corporal, la identidad sexual y las identidades y relaciones familiares de la misma persona, que vienen como consecuencia de esta realidad, como son la maternidad, la paternidad, la filiación, la fraternidad, etc., las cuales pertenecen a un organismo; marcando totalmente la vida de una persona. Por eso, la condición sexual no puede ser una condición irrelevante, sino un camino personal inevitable, muy importante para la búsqueda y formación de la propia identidad (Castilla De Cortazar, 2004).

Sabemos que los factores biológicos no son la totalidad de elementos que conforman la identidad sexual de la persona; por tanto la diferencia entre el varón y la mujer no se limita a la dimensión somática o biológica; la identidad sexual se continua construyendo posteriormente al nacimiento, por etapas durante toda la vida; sin embargo en la etapa de la pubertad la identidad sexual se establece definitivamente, afirmando por esto que la adquisición de la identidad es un proceso psicológico y social, basada en la realidad biológica; habiendo factores diversos que adquieren protagonismo durante la vida personal y social (Aparisi, 2011). La sexualidad es una dimensión constitutiva del ser humano, que impregna profundamente toda la persona y es fundamental para la formación de la propia identidad, como individuo condiciona al hombre como varón o mujer, en su 
desarrollo, en su madurez e integración de los valores corpóreos, psíquicos y espirituales, como también a la sociedad (Lucas Lucas, 2008).

Desde el punto de vista antropológico se tiene dos perspectivas respecto a la identidad sexual: una objetiva y otra subjetiva. Desde la perspectiva objetiva la identidad personal se asume y da poco a poco a través de la toma de conciencia, mientras que la perspectiva subjetiva de la identidad personal se da cuando cada persona organiza los elementos de la realidad desde su originalidad; teniendo como resultado una única y original historia, por ser así la persona (Aparisi, 2011).

\section{Dos modos de ser: masculino y femenino}

Una cuarta directriz, importante es enseñar de forma adecuada y responsable que la persona se manifiesta en dos modos de ser: masculino y femenino. Por tanto los estados intersexuales, 0 el hermafroditismo no son biológicamente un tercer sexo, ya que las personas son cromosómicamente masculinas y femeninas (Elosegui Itxaso, 2011), es decir son varones o son mujeres.

Esta premisa ha tropezado con la ideología de género, que se apoya "en una nueva antropología en una original visión de la persona $y$, más en concreto, de las relaciones entre naturaleza y cultura en la configuración de la identidad humana" (Aparisi, 2014, p. 179), para esta teoría se sostiene que cualquier diferencia entre varón y mujer responde íntegramente a un proceso de socialización e inculturación (Aparisi, 2014) por tanto la identidad sexual no estará configurada por el sexo es decir por lo biológico, lo dado, es decir el ser varón o ser mujer sino que esto es una categoría sin importancia lo que configuraría la identidad sexual para esta teoría será sólo el género, el cual se configura por la influencia de nuestro entorno cultural, de lo que se deriva que podrá existir una multitud de géneros, se podría ser: varón heterosexual, mujer heterosexual, varón homosexual, mujer homosexual, transexual, bisexual, entre otros36. En este sentido se produce una desconfiguración del ser persona, totalmente opuesta a la naturaleza (Aparisi, 2014, p. 179).

\section{Reciprocidad y Complementariedad}

La pregunta por el ser humano, constituye el centro de la filosofía, "se desea saber no solamente qué es el ser humano en abstracto, sino el hombre concreto en su singularidad irrepetible (...). Esta singularidad acoge el cuerpo y acoge el sexo, el ser varón y el ser mujer" (Castilla De Cortazar, 2004, p. 26).

Existe muy poca profundización de la antropología de la sexualidad, se requiere un estudio profundo en los planes de educación de la sexualidad, que aborde lo referente a la masculinidad y feminidad. Pero como refiere Castilla Cortázar, para estudiar el género es necesario partir de dos categorías como son la igualdad y la diferencia39, estas dos categorías deben ser entrelazadas para no caer en el igualitarismo, corriente de pensamiento que busca la equiparación total de mujeres con varones, negando toda diferencia, no viendo en ella la llama riqueza en la diferencia.

A fin de evitar este problema, debe darse el estudio de la reciprocidad y de la complementariedad. En razón de ello, según el Diccionario de la Real Academia de la Lengua Española (RALE), es recíproco aquello igual en la correspondencia mutua, mientras que es complementario lo que sirve para completar o perfeccionar. Por tanto, hablar de reciprocidad 
es tocar el terreno de la igualdad de varón y mujer, mientras que hacerlo sobre complementariedad es abordar la diferencia. A continuación ahondaremos un poco más sobre estas dos categorías.

En cuanto a la persona humana, no es posible estudiar uno al margen del otro, por lo que se hace indispensable recurrir a la igualdad para salvar la riqueza de la diferencia. Por lo que siguiendo a Castilla de Cortázar, podemos establecer que Reciprocidad está orientada a la igualdad en dónde varón y mujer somos iguales, en razón de su dignidad, ninguno es más que el otro. El propio hecho de pertenecer a la especie humana, hace al varón y a la mujer dignos y por tanto iguales. Esta igualdad también permite al varón y a la mujer la posibilidad de correspondencia mutua.

Por otro lado, en razón de la complementariedad, esta encuentra su fundamento en la diferencia. Sosteniendo que: "afirmar que la diferencia varón - mujer es una diferencia en la persona supone por otra parte, haber anclado la diferencia definitivamente en la igualdad. Varón y mujer, cada uno es persona. Tienen la misma categoría; la diferencia entre ellos posee el mismo rango ontológico. La diferencia no rompe la igualdad (Castilla De Cortazar, 2004).

En este sentido Jung advirtió que los sexos no son sólo complementarios entre ellos, sino en el interior de cada uno: y hablaba de que cada varón tiene su ánima - su parte femenina y como contrapartida cada mujer su animus- su parte masculine (JUNG, 1981).

Así el ser varón o el ser mujer son dos modos complementarios y recíprocos de encarnar la misma naturaleza. De tal forma que sus diferencias son imprescindibles en todas las esferas. $Y$ sus peculiaridades son relacionales, complementarias y recíprocas, cada uno se apoya en el otro, cada uno encuentra su posibilidad en el otro. Se podría decir por tanto que no existen espacio social femenino o espacio social masculino, pues en todas las esferas (laboral, familiar, político) pueden y deben estar presentes ambos (Castilla De Cortazar, 2004).

\section{La dimensión de apertura en el varón y en la mujer}

Si debemos unir la condición sexuada con persona, ha de ser en su dimensión de apertura. Cada persona se abre para amar, pero se puede abrir de un modo diferente y complementario, esto es lo que ocurre entre el varón y la mujer.

Ahora la apertura constitutiva que tiene cada persona tiene dos modalidades: el varón se abre de un modo peculiar: hacia afuera. La mujer se abre a los demás con su modo: hacia dentro acogiendo. El varón al darse sale de sí mismo. Saliendo de él se entrega a la mujer y se queda en ella. La mujer se da pero sin salir de ella. Es apertura pero acogiendo en ella. Su modo de darse es distinto al del varón y a la vez complementario, pues acoge al amor y a su amor. Sin la mujer el varón no tendría donde ir. Sin el varón la mujer no tendría qué acoger. La mujer acoge el fruto de la aportación de los dos y lo guarda hasta que germine y se desarrolle. Todo este proceso, aunque él es también protagonista, se realiza fuera del varón (Castilla De Cortazar, 2004). Al varón le corresponde la preposición DESDE, pues parte de sí para darse a los demás. La mujer le corresponde la preposición EN: pues se abre dando acogida en sí misma. La persona varón se podría describir, entonces con SER - CON - DESDE, o COEXISTENCIA - EN (Castilla De Cortazar, 2004). 
Este modo de darse diferente y complementario se da en todos los campos y en todas las relaciones humanas heterosexuales, y apoyándose en la dimensión constitutiva de apertura que el ser humano posee y que da como resultado: la persona femenina y la persona masculina. De allí que la diferencia sexual se trataría de una diferencia en el mismo interior del ser. $Y$ teniendo en cuenta que el ser humano es personal, sería una diferencia en el seno mismo de la persona (Castilla De Cortazar, 2004).

\section{Conclusiones}

La educación de la sexualidad se concreta a través de dos presupuestos: la educación de la castidad y el enfoque de la complementariedad o la integridad.

Ante la necesidad de la educación de la sexualidad se deben tener en cuenta las siguientes directrices de salud para implementar programas de educación de la sexualidad: visión integral de la persona y su sexualidad; la persona es cuerpo y espíritu; importancia de una adecuada educación de la identidad sexual; dos modos de ser: masculino y femenino; reciprocidad y complementariedad y la dimensión de apertura en el varón y en la mujer.

\section{Bibliografía}

APARISI, A. (2009). La ideología de género: de la naturaleza a la cultura. Persona $y$ Derecho.

APARISI MIRALLES, A. (2011). Persona y género: ideología y realidad. Pamplona, España: Aranzadi.
APARISI MIRALLES, Á. y BALLESTEROS, J. (2002). Por un feminismo de la complementariedad: Nuevas perspectivas para la familia y el trabajo. Pamplona, España: Ediciones Universidad de Navarra S.A.

BARBOZA ZAPATA, E. El hombre unidad substancial de cuerpo y alma. [Ubicado el 8.VII.2016]. Recuperado de https://es.scribd.com/doc/6420348/ElHombre-Unidad- Substancial-Del-CuerpoHumano

BELTRAMO ÁlVAREZ C. E. Fundamentos Antropológicos de la Sexualidad Humana. [Ubicado el 8.VII.2016]. Recuperado de

http://www.mercaba.org/FICHAS/Alafa_or g/educacion_sexual2.htm

BURGOS, Juan Manuel. (2011). Dos formas de afrontar la identidad sexual: personalismo e ideología de género. Persona y género: ideología y realidad. Pamplona. España: Editorial Aranzadi.

CAFARRA, C. (2002). Sexualidad a la luz de la antropología y la biblia, Documentos del Instituto de Ciencias para la familia. Madrid, España: Ediciones RIALP.

CASTILLA DE CORTAZAR, B. (2004). La complementariedad varón -mujer: nuevas hipótesis. Instituto de Ciencias para la familia. Madrid, España: Ediciones RIALP. S.A.

CASTILLA DE CORTAZAR, B. (2004). Persona femenina, persona masculina. Instituto de ciencias para la familia. Madrid, Esáña: Ediciones Universidad de Navarra. 
DE IRALA, J. (2012). El valor de la espera. Madrid, España: Ediciones Palabra.

ELOSEGUI ITXASO, M. (2011). Tres diversos modelos filosóficos sobre relación entre sexo y género. Persona y género: ideología y realidad. Pamplona, España: Editorial Aranzadi.

GARCÍA HOZ, V. (2002). Educación de la sexualidad. Madrid, España: Ediciones RIALP S.A.

HERVADA, J. (1991). Los derechos inherentes a la dignidad de la persona humana. Humana lura.

JUAN PABLO II. (1988). Carta Apostólica Mulieris dignitatem. 15. VIII.

JUNG, C. (1981). Los arquetipos y el concepto de "anima". Arquetipos e inconsciente colectivo.

LUCAS LUCAS, R. (2005). Explícame la bioética: Guía explicativa de los temas más controvertidos sobre la vida humana. Madrid, España: Editorial Palabra.

LUCAS LUCAS, R. (2008). Horizonte vertical sentido y significado de la persona humana. Madrid, España: Estudios y ensayos, Biblioteca de autores cristianos.

MINISTERIO DE SALUD (MINSA), Situación de la salud sexual y Reproductiva en el Perú, 2014. [Ubicado el 18.XII 2014]. Recuperado de $\mathrm{ftp}: / / \mathrm{ftp} 2 . \mathrm{minsa} . g o b . p e /$ descargas/03esn/1 2ESN_sexual/SSR_PERU.pdf

ORGANIZACIÓN DE LAS NACIONES UNIDAS PARA LA EDUCACIÓN DE LA CIENCIA Y LA
CULTURA (UNESCO), Orientaciones Técnicas Internacionales sobre educación en sexualidad- Un enfoque basado en evidencias orientadas a escuelas docentes y educadores de la Salud, Junio 2010 [Ubicado el 17.XII.2014]. Recuperado de http://unesdoc.unesco.org/images/0018/0 01832/183281s.pdf

POLO, L. (1897). Tener y dar. Estudios sobre la Encíclica 'Laborem exercens'. Madrid, BAC.

SARMIENTO, A. La integración de la sexualidad en el bien de la persona (consideración en torno a algunos "problemas)» [Ubicado el 24.II 2016]. Recuperado de file:///C:/Users/Kelly/Downloads/ST_XXXI3_02\%20(1).pdf

SARMIENTO, A. Persona, sexualidad humana y procreación. p. 387. [Ubicado el 20.1 2016]. Recuperado de http://dadun.unav.edu/bitstream/10171/5 335/1/AUGUSTO\%20SARMIENTO.pdf 